\title{
APPLICATION OF AN AUTONOMOUS ROBOT FOR THE COLLECTION OF NEARSHORE TOPOGRAPHIC AND HYDRODYNAMIC MEASUREMENTS
}

\author{
Frauke Wübbold ${ }^{1}$, Matthias Hentschel ${ }^{1}$, Michalis I. Vousdoukas ${ }^{2}$ and Bernardo Wagner ${ }^{1}$ \\ Beach topographic and hydrodynamic measurements are essential for coastal geology and engineering studies as well \\ as sustainable coastal management. Standard approaches involve either time-consuming manual data acquisition \\ usually with limited coverage or remote sensing techniques which are usually characterized by low resolution or \\ increased costs. The present contribution reports the results from the application of the autonomous robot RTS- \\ Hanna with a calibrated sensor setup including 3D laser range scanners, a camera, a Differential GPS and an inertial \\ measurement unit which significantly facilitates field data collection. RTS-Hanna was tested at the Wadden Sea \\ Barrier Island Langeoog, Northern Germany, for two days and was proven capable of autonomously collecting \\ topographic scans. $175 \mathrm{~GB}$ of dense topographic and water surface elevation data were collected, including RBG \\ images, while RTS-Hanna covered a total of $21 \mathrm{~km}$ of coastline in approximately 3 hours. Scans of the surf/swash \\ zone allowed continuous measurements of topographic changes at the beachface, wave propagation velocities and \\ wave breaking heights.
}

Keywords: remote sensing, 3D laser range scanner, topographic measurements, hydrodynamic measurements, autonomous robot.

\section{INTRODUCTION}

The temporal response of beaches can be considered at instantaneous to millennial scales, while the spatial scales of beach systems range from $\mathrm{mm}$ to several $\mathrm{km}$, from sediment grains to individual beaches and coastlines (De Vriend 1991), requiring intensive data acquisition schemes. On the other hand, data acquisition in the nearshore zone comes with several challenges like the high spatial variability requiring dense grids of measuring points and the highly energetic conditions which often prohibit or increase risk and difficulty of in-situ data collection. All methods mentioned above make non-intrusive approaches like coastal video monitoring stations (Holman and Stanley 2007, Vousdoukas et al. 2011a) and X-band radar systems (Senet et al. 2008) very attractive or even indispensable. Such systems are ideal for long-term deployments (i.e. years) because they acquiring data continuously, covering several hundreds of metres of coastline and allowing the study of nearshore processes at various temporal and spatial scales. However, they usually come with the drawback of reduced accuracy.

For more accurate field topographic measurements, off-road vehicles (e.g. ATVs) equipped with Differential Geographical Positioning Systems (D-GPS) are being commonly used in order to increase the time efficiency and spatial coverage, whereas the man-hours and costs for frequent (e.g. daily/weekly) surveys still remain high. Satellite images lack the temporal resolution, a disadvantage that also applies to the expensive airborne LIDAR surveys. Small Unmanned Aerial Vehicles (Vousdoukas et al. 2011b) for coastal surveys are able to cover wide areas yet entailing several drawbacks such as that they have to be launched manually, thus requiring personnel, and the incapacity to carry heavy laser scanning equipment, compromising the accuracy of the measurements. Thus, Terrestrial 3D Laser Scanning (TLS) has increasingly become the method of choice for beach surveying (e.g. Pietro et al. 2008, van Gaalen et al. 2011). Portable scanners allow the completion of beach surveys in excess of hundreds of metres with sub-centimetre spatial resolution, over the course of a few hours. These advances provide a reliable means of addressing geomorphic relationships as well as along- and cross-shore changes on the beach, while the same accuracy and spatial resolution is impossible to be achieved with traditional D-GPS surveys. The technique has been introduced quite recently in coastal research and offers several unexploited possibilities, e.g. for wave measurements (Park et al. 2011). Still, trained personnel are required and costs related to daily measurements remain high.

Against the foregoing background, the present contribution sets out to present a new approach to collect high resolution/accuracy topographic measurements for wide coastal stretches (i.e. several km), using an autonomous robotic platform equipped with a wide array of sensors. Testing took place at a

\footnotetext{
${ }^{1}$ Real Time Systems Group, Institute of Systems Engineering, Leibniz Universität Hannover, Appelstraße 9A, 30167 Hannover, Germany

${ }^{2}$ Forschungszentrum Küste, Leibniz Universität Hannover, Merkurstraße 11, 30419 Hannover, Germany
} 
Barrier Island located and the East Wadden Sea and the results presented in the following sections make very interesting reading.

\section{AUTONOMOUS MEASUREMENT ROBOT RTS-HANNA}

The autonomous robot RTS-Hanna (Fig. 1) is based on a car-like Kawasaki Mule 3010 side-byside vehicle powered by a $24 \mathrm{HP}$ diesel engine which enables a top speed of $40 \mathrm{~km} / \mathrm{h}$. The robotic platform has an overall length of $285 \mathrm{~cm}$, a width of $144 \mathrm{~cm}$ and a total weight of about $800 \mathrm{~kg}$ while tolerating a payload of up to $600 \mathrm{~kg}$. The vehicle is equipped with differential-locks and four-wheeldrive and thus is well suited for rough environments like coastal areas. The vehicle is fully street licensed and allows for either manual control or full computer control via CAN-BUS due to a drive-bywire system from PARAVAN GmbH. The sensor system includes four 3D laser scanners that work on the time-of-flight measurement principle, a camera, D-GPS and inertial sensors.

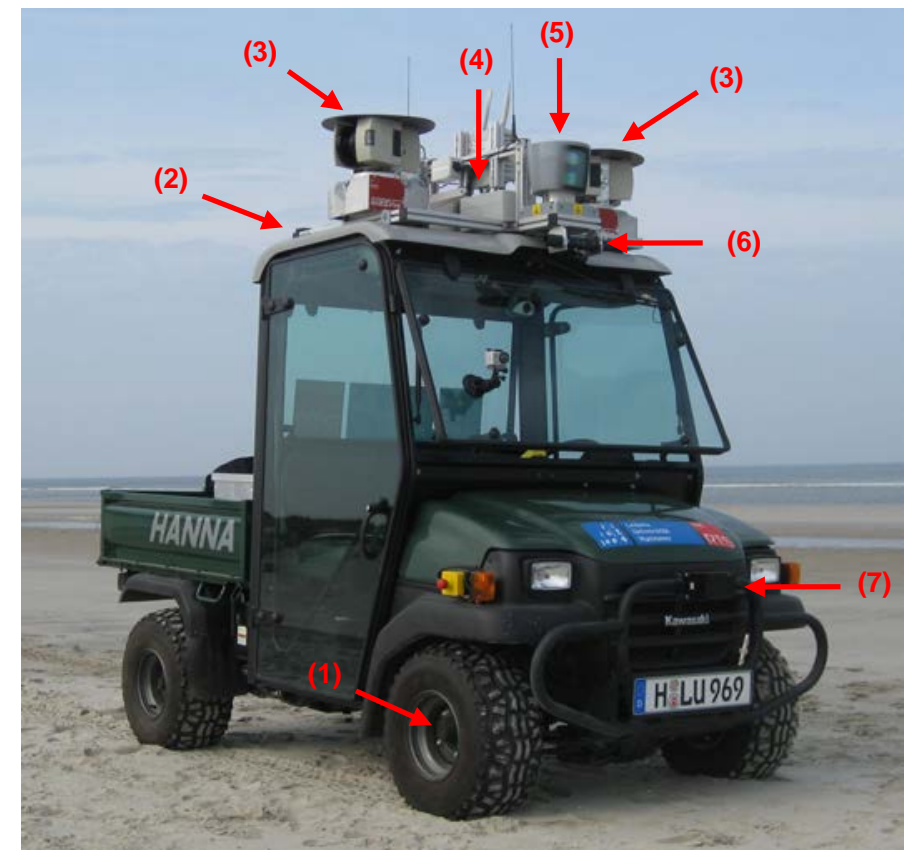

Figure 1. Autonomous robot RTS-Hanna: (1) wheel odometry, (2) D-GPS Trimble AgGps 114, (3) ScanDrive, (4) Gyro PerformTech Gu3024, (5) Velodyne HDL-64E, (6) Microsoft Kinect, and (7) ibeo LUX.

Two of the laser range scanners are RTS-ScanDrives each consisting of two SICK LMS 291 mounted vertically on a rotational unit and operating at $0.8 \mathrm{~Hz}$ with a distance accuracy of $+/-5 \mathrm{~cm}$. They have a maximum range $30 \mathrm{~m}$ and are used for near-field perception as they provide a full $360^{\circ}$ by $181^{\circ}$ field of view with an angular resolution of $1^{\circ}$ in vertical and $2^{\circ}$ in horizontal direction. The third laser range scanner is a commercial Velodyne HDL-64E with a field of view of $360^{\circ}$ by $26.8^{\circ}$, a maximum range of up to $120 \mathrm{~m}$ and a distance accuracy of $<5 \mathrm{~cm}$. It provides an angular resolution of $0.4^{\circ}$ vertically and $0.09^{\circ}$ horizontally, a distance resolution below $5 \mathrm{~cm}$, and operates at $15 \mathrm{~Hz}$. The fourth 3D laser scanner is an ibeo LUX with four horizontal parallel scanning layers with a vertical angular distance of $0.8^{\circ}$ and a horizontal resolution of $0.25^{\circ}$ when operating at $25 \mathrm{~Hz}$. Detecting up to 3 reflections per laser impulse, the ibeo LUX is able to measure through atmospheric clutter like dust or moderate rain and to pre-classify measurement into ground, clutter/rain/dust, and valid/obstacle. This scanner is mainly used for fast obstacle detection in front of the robot. The camera mounted on RTSHanna is a Microsoft Kinect operated with an image size of 640 by 480 pixels and at $10 \mathrm{~Hz}$. For position estimation, the robot is equipped with wheel encoders providing an update rate of $10 \mathrm{~Hz}$ and a Trimble AgGPS 114 with sub-metre accuracy due to Omnistar differential corrections working at $1 \mathrm{~Hz}$. The inertial measurement unit (IMU) is a fibre optic gyroscope PerformTech Gu3024 which operates at $60 \mathrm{~Hz}$ and provides the accelerations along the three Cartesian axes as well as angular velocities around these axes.

Data acquisition and processing is performed by five Embedded PCs operating at $700 \mathrm{MHz}$ to 2.16 $\mathrm{GHz}$ onboard the robot. They conduct real-time data processing and run the operating system LiRE (Linux Real-time Environment, http://www.rts.uni-hannover.de/lire) which contains the real-time 
extension Xenomai and the hard real-time protocol stack RTNet (http://www.rtnet.org). The robotic functionality, i.e. measuring, autonomous operation, data logging and re-playing, is provided by the Robotics Applications Constructions Kit RACK (http://developer.berlios.de/projects/rack), which is a middleware developed and permanently refined by the Real Time Systems Group and large parts of which are open-source.

\section{ROBOTIC FUNCTIONALITY}

\section{Autonomous Functions}

For driving autonomously to a given destination, the robot requires specific information about its surroundings as well as its task which can be boiled down to four questions each covering a different area of robotic:

1. Where am I?

2. What's my environment like?

3. Where should I go?

4. How do I get there?

Self-localisation

Obstacle and landmark detection

Path planning

Movement control

An overview of the data processing required for an autonomous robot like RTS-Hanna is given in Fig. 2. In addition to the autonomous functionality, the RACK provides functions for data logging and replaying, including a robot simulation. This significantly simplifies evaluation and re-processing of raw data, e.g. with a different strategy for obstacle detection, or additional post-processing.

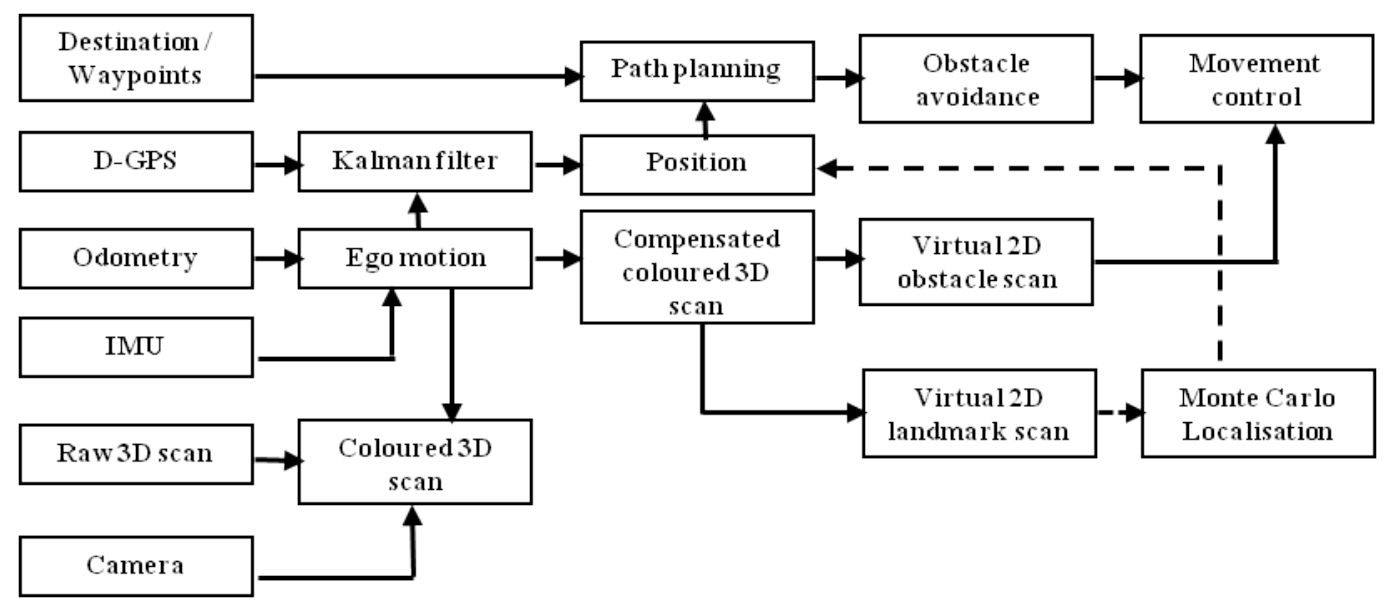

Figure 2. Data processing for environment perception and autonomous navigation. Dashed lines indicate more sophisticated self-localisation with MCL.

\section{Self-localisation and ego-motion compensation}

For ego-motion estimation, movement increments are measured by the wheel encoders and subsequently fused with angular velocity data provided by the inertial measurement unit. By integration over time, the 6DoF robot position, consisting of position and orientation, is available. This global robot position is improved by Kalman-filtered fusion with the D-GPS data. A Kalman filter estimates the exact value which underlies a series of noisy measurement data by assuming Gaussian noise and using a Bayesian model. The resulting 6DoF robot position is given in e.g. the WGS84 system and enables the transformation to a global system for all data given in the robot-fix Cartesian system, which is used for local data processing like dynamic obstacle information. The robot position counts as selflocalisation and could further be improved with methods relying on given environmental knowledge like the Monte-Carlo-Localisation (MCL, Dellaert et al. 1999).

A 3D laser range scanner collects distance data relative to a spherical coordinate system which is fix relative to the scanner. For further processing, the distance information has to be transformed into a robot-fix Cartesian system, the horizontal plane of which corresponds to the WGS84 horizontal plane with a height offset, therefore compensating for the robot's ego motion. This includes the angular displacements, e.g. when the robot is tilted at sloped ground as well as the robot's motion during a single 3D laser range scan. A raw (a) and compensated (b) stationary Velodyne HDL-64E scan is depicted in Fig. 3, taken at the beach while facing the water (c). The scan points are coloured according to their elevation. 


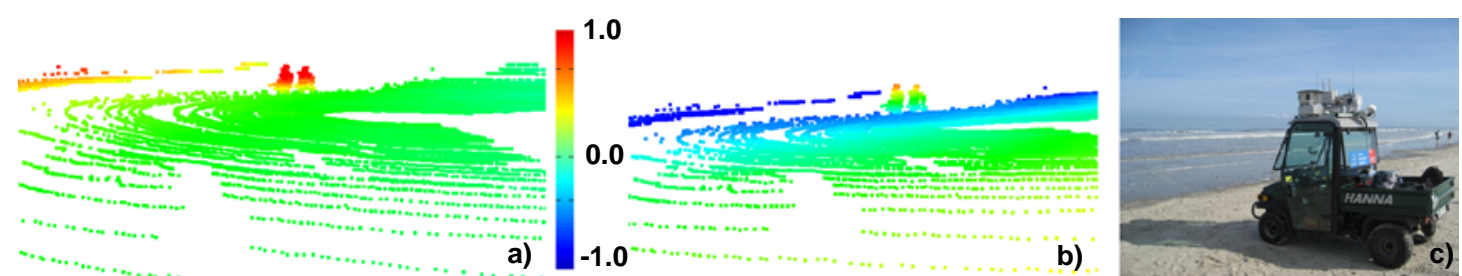

Figure 3. Raw (a) and compensated (b) stationary Velodyne HDL-64E scan of the scene (c) with two squatting persons. The scan points are coloured according to their elevation with a metrical scale with zero at the ground level beneath the robot.

\section{Landmark and obstacle detection with Virtual 2D Scans}

Landmark and obstacle information are essential for autonomous driving. The former enables collision avoidance; the latter is used for a more precise and reliable self-localisation. By creating a Virtual 2D Landmark Scan or a Virtual 2D Obstacle Scan (Wulf et al. 2004), this information is extracted from the 3D point cloud resulting in significantly reduced data and enabling 2D localisation or 2D collision avoidance methods, respectively, which are nearly as detailed as but much faster than their 3D equivalents. Landmarks are specific static structures in the robot's environment which can be detected robustly and thus are feasible for being matched to a given map in order to identify the robot's position. In urban areas, landmarks typically are walls of buildings.

For landmark, i.e. wall, detection, the 3D laser range scan is transformed into a range image. The scan points are organized in rows and columns according to their vertical and horizontal angle steps and assigned a gray-level according to their distance. Horizontal and vertical edge detection with subsequent flood-filling identifies flat surfaces. An additional object-ground classification separates flat ground from wall surface. It applies the heuristic that a scan point is an object point if there is at least another scan point in a cone below it. Simultaneously, it identifies which scan points most likely belong to the same real-world object by clustering all points which lie in each others' cones. The clusters are called segments. Fig. 4 shows the segments obtained for a group of beach baskets, where light yellow indicates segment points, red visualises segment edges and green all points classified as ground.
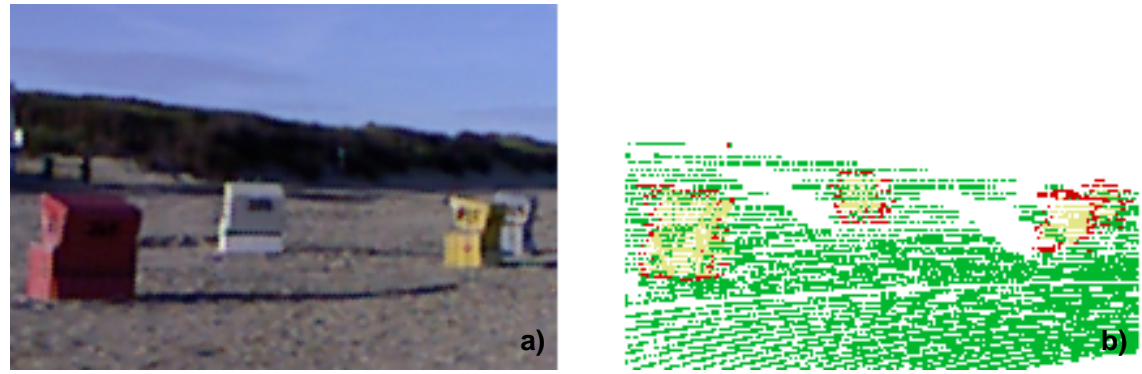

Figure 4. Scene with beach baskets (a) and segmented scan (b) with segments (light yellow). Segment edges are coloured in Red and points classified as ground in Green.

In case there is more than one "wall surface” point per horizontal angle step, the most distant landmark point is chosen for the Virtual 2D Landmark Scan in order to make it more robust to clutter caused for example by smaller objects in front of a wall. The smaller objects might be semi-static or dynamic obstacles and thus not relevant for localisation; or they are static and thus could be used as landmarks but they lack of detection robustness due to their small size and thus are not element of the given map.

Obstacle points are scan points with an elevation above the ground which does not allow the robot to safely drive above or below. The most relevant obstacles are the ones closest to the robot, i.e. obstacles in the shadow of closer ones can be neglected. The Virtual 2D Obstacle Scan exploits this idea and extracts only the nearest scan point satisfying the potential collision height interval for each horizontal angle step.

While a full 3D laser range scan consists of up to 32000 points (RTS-ScanDrive) or up to 268000 points (Velodyne HDL-64E), a Virtual 2D Scan contains all essential information on either landmarks or obstacles with at most 180 points for the RTS-ScanDrive or 4000 points in case of the Velodyne HDL-64E. 


\section{Path-planning and movement control}

RTS-Hanna is able to travel autonomously from its known initial position to a given global destination. A continuous path is generated based on spline mathematics and takes into account the kinematic constraints of the vehicle like maximum speed, maximum acceleration and minimum curve radius. In a more complex environment, RTS-Hanna can be given an additional set of waypoints in order to facilitate path planning e.g. at intersections. The waypoints are defined by the operator either prior to the navigation task or on the fly in a Graphical User Interface. A path along a set of waypoints is a spline which continuously connects them. A cascade controller which combines feedforward and feedback control for high accuracy enables fully autonomous driving along the generated path. Following the path involves reaction to obstacles, either by stopping in front of the obstacle and waiting until the path is clear, or by avoiding the obstacle and therefore travelling on new path past the obstacle and back to the original path as soon as possible. The desired reaction to obstacles can be chosen at run time. For obstacle avoidance, additional restrictions like the maximum lateral deviation from the originally planned path are available, which facilitates e.g. autonomous driving in the same area with multiple robots.

\section{Coloured 3D Scan}

In front of the robot, the fields of view of the 3D laser range scanners and the camera overlap. Thus for the given calibrated setup, corresponding pairs of scan point and camera pixel can be identified. A top view of a set of coloured scans merged together according to their GPS position is shown in Fig. 5.

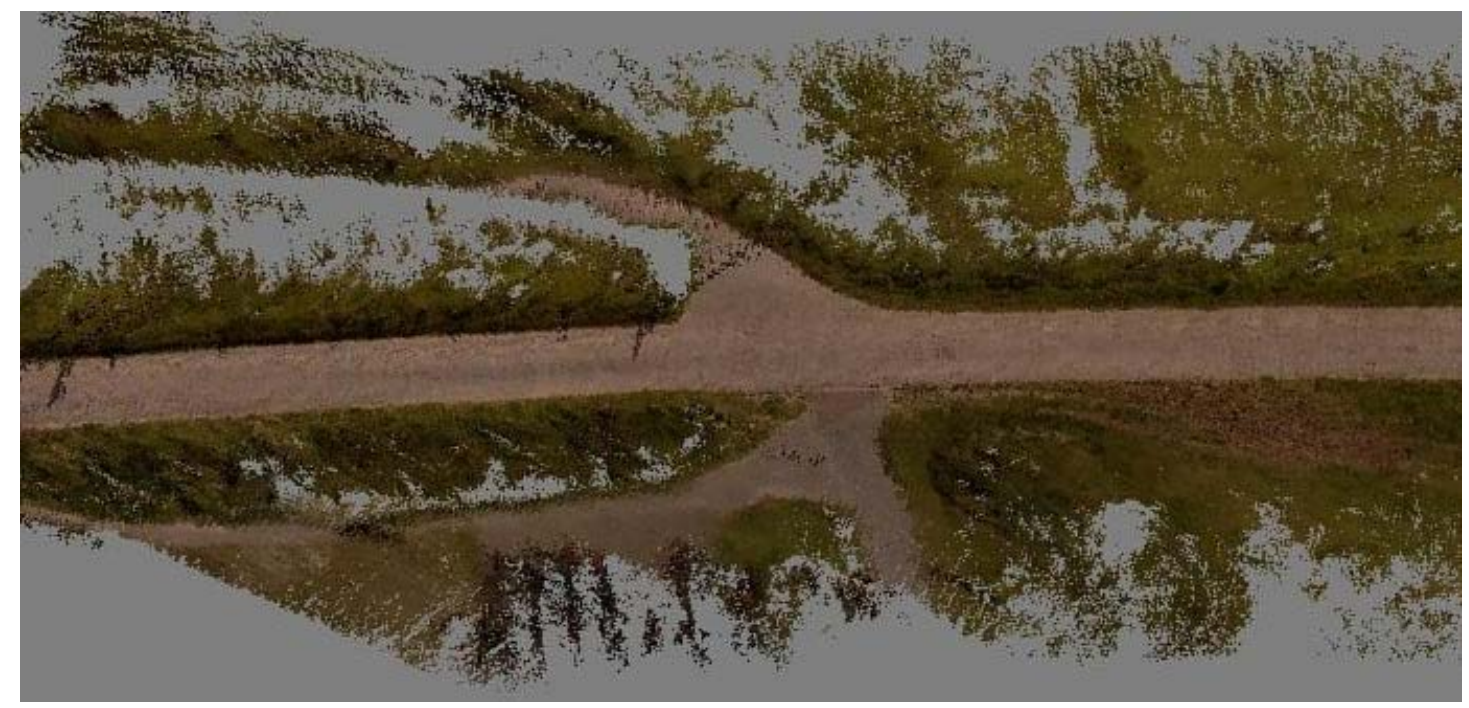

Figure 5. Coloured 3D scans merged by D-GPS position, viewed from the top, with parts invisible to the scanner displayed in Grey.

\section{FIELD TESTING}

\section{Testing site}

Testing took place at Langeoog, part of the East Frisian Islands, a high-touristic value site with $\sim 2000$ habitants concentrated mostly at the western part (Fig. 6 b). The area is meso-tidal with tidal range varying from $\sim 2 \mathrm{~m}$ (neaps) to $\sim 3 \mathrm{~m}$ (springs), with a yearly mean offshore significant wave height around $H_{s} \sim 1 \mathrm{~m}$ and waves mainly approaching from western to northwestern directions (Niemeyer 1992). Storm surge is a very important erosion/hazard element exceeding $3 \mathrm{~m}$ during extreme events (www.wsv.de); while sediments in the area are fine sands (classification according to Folk 1980), with $d_{50}$ between $0.2 \mathrm{~mm}$ and $0.225 \mathrm{~mm}$ (Ehlers, 1988).

Similarly to other Frisian Islands, Langeoog has been shown to gradually migrate mainly to the East but also to the South (Ehlers 1988, Flemming and Bartholomä 2003). As a result the dunes and the beach in front of the human settlement have been undergoing erosion over the last decade with a recorded annual dune retreat rate exceeding $10 \mathrm{~m}$. Apart from the socio-economically highly interesting shoreline dynamics and erosion/recovery processes, unique nearshore bar features and behaviour have been observed in the area. Ehlers (1988) reported the presence of 'saw-tooth' sandbars which are very 
dynamic and have been shown not only to undergo several shape changes, but also to detach from the East Baltrum coast gradually welding to the West Langeoog coast (Fig. 6 b).
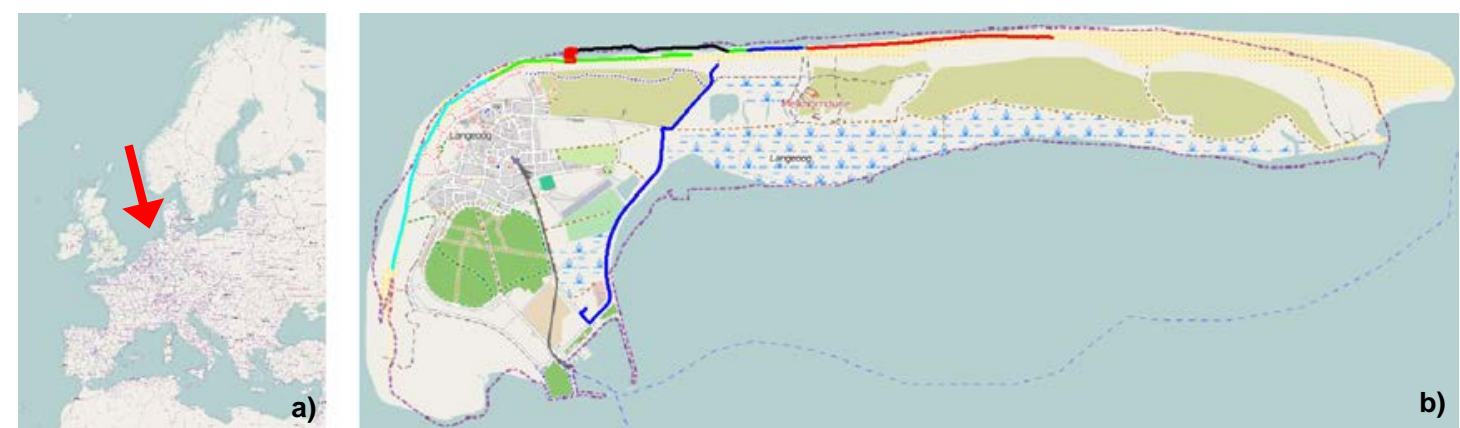

Figure 6. Study area in the Northern Germany, OpenStreetMap (a); paths of RTS-Hanna during the data acquisition superimposed with the OpenStreetMap data of Langeoog, runs displayed in different colours (b).

\section{Testing approach}

The field campaign took place at the North Frisian barrier island Langeoog in Northern Germany on September $26^{\text {th }}$ and $27^{\text {th }} 2011$ and aimed at evaluating the benefits and drawbacks of a measurement vehicle like RTS-Hanna, equipped with a calibrated sensor setup and able of autonomous driving and data collection. Intending to collect near-shore topographic data, the measurement runs were conducted during low tide at dry beach testing different surveying strategies. Most of these runs contained measurements of a dune partially covered with tall grass as well as small wind-generated sand dunes of about $20 \mathrm{~cm}$ height. Beach visitors, beach baskets and sporting grounds were present in a few runs. An additional stationary monitoring run of the surf and swash zone provided colour information of the water and beach surface as well as distance information of the beach surface, the breaking and propagation of waves and swash motions. In total, a beach area of $21 \mathrm{~km}$ was covered in approximately 3 hours resulting in $175 \mathrm{~GB}$ of raw data, while travelling at an average velocity of $10 \mathrm{~km} / \mathrm{h}$. Fig. 6 shows the OpenStreetMap (OSM, http://www.openstreetmap.org) data of the test site, the close-up view in (b) superimposed with the path for each run. Table 1 gives an overview of the travelled distances, average velocities and the most relevant collected data. For coastal laser range data acquisition, the Velodyne HDL-64E provided the most interesting information as it is much more detailed than that of the low-resolution ScanDrives. Nonetheless, ScanDrive data with its $181^{\circ}$ vertical field of view is essential for autonomous navigation as it covers the area in the immediate vicinity of the robot.

\begin{tabular}{|c|c|c|c|c|c|c|}
\hline \multirow[t]{2}{*}{ Run } & \multirow{2}{*}{$\begin{array}{l}\text { Distance } \\
{[\mathrm{m}]}\end{array}$} & \multirow{2}{*}{$\begin{array}{l}\text { Duration } \\
\text { [s] }\end{array}$} & \multicolumn{2}{|c|}{ Number Images / Scans } & \multicolumn{2}{|c|}{ Sizes Images / Scans [MB] } \\
\hline & & & Kinect & Velodyne & Kinect & Velodyne \\
\hline 1 & 3579 & 1577 & 28233 & 19392 & 24814.16 & 5397.15 \\
\hline 2 & 896 & 613 & 10976 & 7500 & 9646.88 & 2087.39 \\
\hline 3 & 866 & 494 & 8401 & 5746 & 7383.69 & 1599.22 \\
\hline 4 & 857 & 387 & 0 & 0 & 0.0 & 0.0 \\
\hline 5 & 606 & 968 & 0 & 0 & 0.0 & 0.0 \\
\hline 6 & 1745 & 793 & 14053 & 9557 & 12351.27 & 2659.89 \\
\hline 7 & 686 & 692 & 14805 & 10087 & 13012.21 & 2807.40 \\
\hline 8 & 2295 & 1016 & 8919 & 12386 & 7838.96 & 3447.25 \\
\hline 9 & 2429 & 1184 & 11151 & 15402 & 9800.68 & 4286.66 \\
\hline 10 & 0 & 696 & 6561 & 9110 & 5766.50 & 2535.48 \\
\hline 11 & 3641 & 975 & 9307 & 12884 & 8179.98 & 3585.85 \\
\hline Sum & 17601 & 9395 & 112406 & 102064 & 98794.34 & 28406.29 \\
\hline
\end{tabular}

Run1 - Long distance: The first measurement was a long distance test travelling $3.5 \mathrm{~km}$ along the northern coast in western direction, starting at the border of the nature reserve area, while keeping a distance of a few ten metres to the shoreline at the right-hand-side and a larger distance to the dune at the left.

Run $2-\mathbf{1}^{\text {st }}$ repetition (of a part of run1): A $0.9 \mathrm{~km}$ length part of the run 1 track was chosen for multiple repetition of the same track in order to get multiple data sets covering the same area. In this run, the robot moved eastward while automatically generating waypoints for the first autonomous measurement in the $4^{\text {th }}$ run. 
Run $3-2^{\text {nd }}$ repetition: While driving along the path of run 2 in western direction, the robot generated waypoints for the second autonomous run (run 5).

Run 4 - Autonomous driving and $3^{\text {rd }}$ repetition: RTS-Hanna autonomously planned and travelled along an eastward path through the waypoints of run 2. At the autonomous driving tests Velodyne HDL-64E data was intentionally not logged as the limited processing units had to perform additional tasks for the autonomous functionality in hard real-time.

Run 5 - Autonomous driving and $4^{\text {th }}$ repetition: RTS-Hanna autonomously planned and travelled along a westward path through the waypoints of run 3 , again intentionally not logging Velodyne HDL-64E data.

Run 6 - Lower intertidal area: Data acquisition along a path at the water/dry beach border, including wet ground and very shallow water (of less than $5 \mathrm{~cm}$ depth) while travelling in western direction covering $1.7 \mathrm{~km}$.

Run 7 - Meandering stop and go: This run covered a more squarely shaped area in meandering fashion stopping at regular intervals in order to collect stationary topographic beach data over a few minutes and thus to apply a method similar to standard stationary TLS scanning.

Run 8 - Long distance at a more populated coastal stretch: In the first run on September $27^{\text {th }}$, RTS-Hanna travelled westwards for about $2.3 \mathrm{~km}$ through an area mostly populated by beach visitors and beach baskets.

Run 9 - Long distance at a more populated coastal stretch: The western coast section of Langeoog was covered in a long distance run $(2.4 \mathrm{~km})$ driving in southern direction at the more populated western coast of Langeoog with several beach visitors, a lot of beach baskets, a swimmer rescue centre and beach volleyball fields.

Run 10 - Stationary hydrodynamic: For collecting Velodyne HDL-64E and Kinect data of uprunning waves, the robot was positioned facing the sea in a distance of a few metres to the waterline.

Run 12 - Long distance inner-island: The last run involved inner-island travelling along a $3.6 \mathrm{~km}$ track from the northern coast to the harbour passing through dunes, along the dune and several buildings.

\section{TOPOGRAPHIC DATA}

\section{Comparison of ScanDrive and Veldoyne HDL-64E}

Low resolution and low frequency ScanDrives provided distance measurements in a full $360^{\circ}$ by $181^{\circ}$ field of view with a maximum range of $30 \mathrm{~m}$. The field of view is important for autonomous functionality as it supplies the robot with obstacle information in its direct vicinity, while the low resolution facilitates data processing in real-time. However, for the collection of topographic data a vertically narrower field like that of the Velodyne HDL-64E does not result in disadvantages. The blind spot surrounding the robot can easily be filled with data from a different nearby position due to the constantly moving robot. The higher resolution and the higher maximum range of up to $120 \mathrm{~m}$ make the Velodyne HDL-64E more suitable for detailed topographic data collection. For example, small windgenerated sand dunes are clearly visible in the Velodyne HDL-64E scans as shown in the left of Fig. 7. Hence the present contribution focused on the high-resolution scan data.

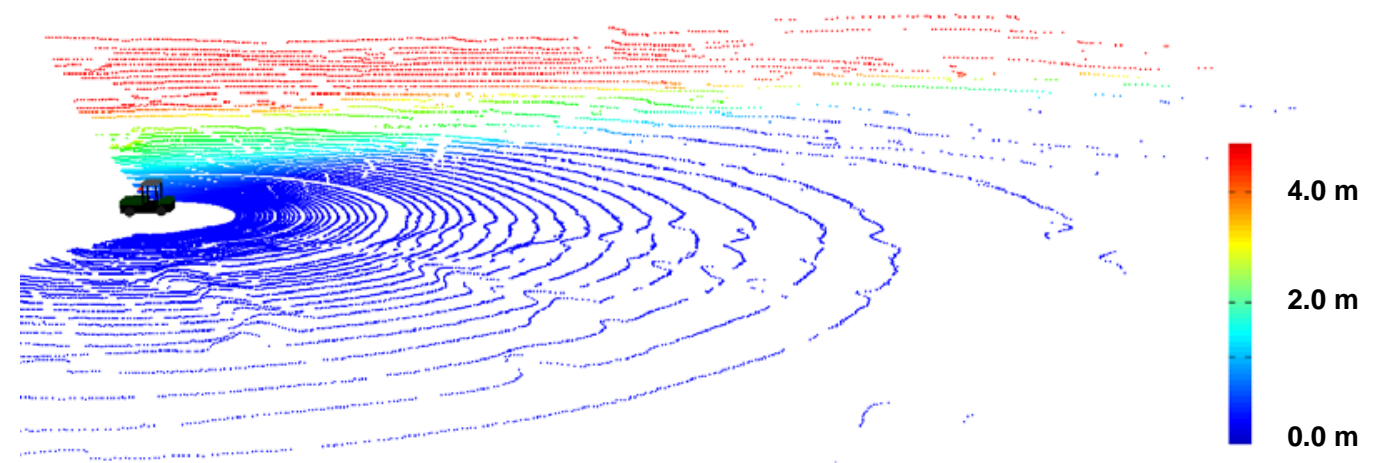

Figure 7. Velodyne HDL-64E scan of a beach section with small wind-generated and the bigger dune coloured by elevation. 


\section{Topographic grid map}

Approximately $10^{\wedge} 10$ 3D scan points collected by the Velodyne HDL-64E data resulted in a Dense Elevation Model covering all surveyed parts of the beach and including the inner-island path from the beach to the harbour. The transformation from the local sensor coordinates to the UTM system (in projection on WGS84) utilized the D-GPS robot position only, which resulted in more reliable data due to erroneous odometry induced by wheel slippage on the sand. Having pre-computed the coordinates of grid points in a distance of $1 \mathrm{~m}$, the height of each point was calculated from the scan points in a $10 \mathrm{~cm}$ $\mathrm{x} 10 \mathrm{~cm}$ neighbourhood of each grid point by averaging. Exemplarily, parts of the overall DEM generated from the data of runs 1, 8 and 11 are depicted in Fig. 8. As clear water surfaces reflect the laser impulses in a specular fashion, the sensor did not detect any return values therefrom.

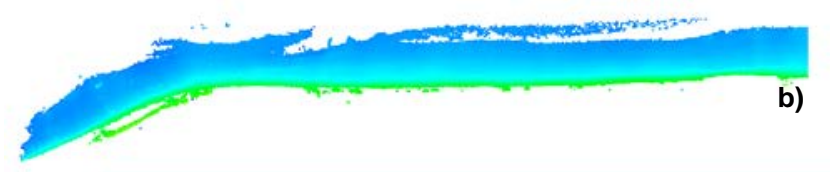

b)

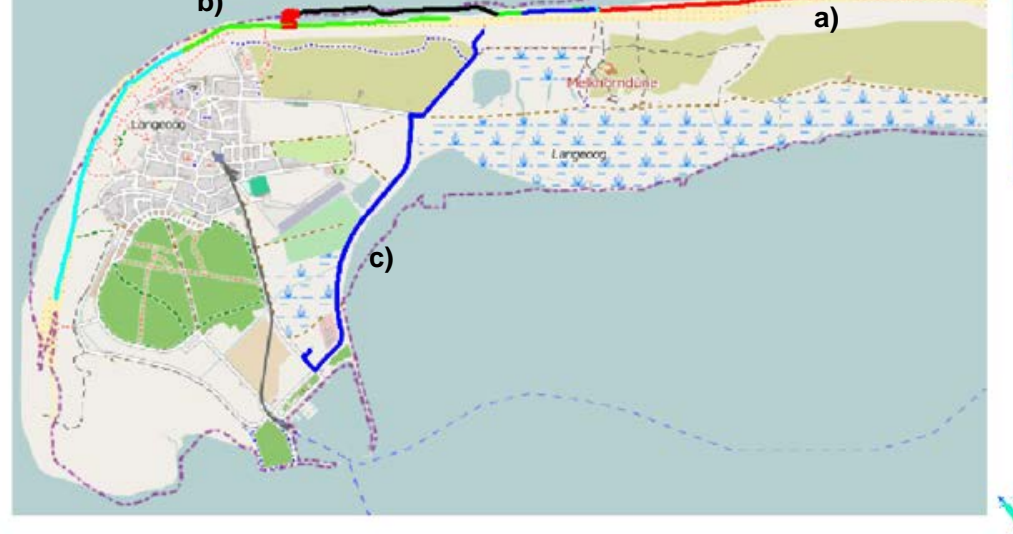

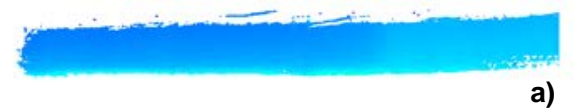

a)

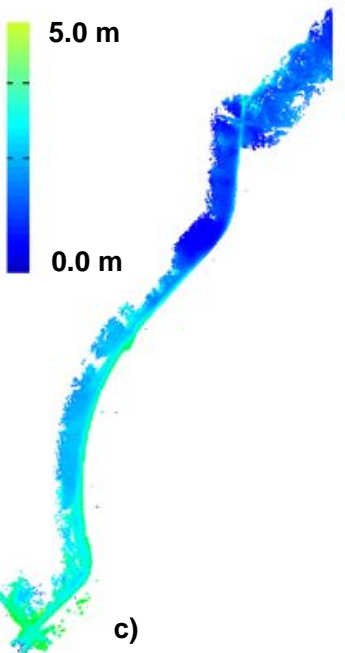

c)

Figure 8. Digital Elevation Models of runs 1 (a), 8 (b) and 11 (c) with OSM map of the testing site.

\section{HYDRODYNAMIC DATA}

Run 11 generated continuous measurements of the water and dry-beach level variations. In conformity with previous studies (Blenkinsopp et al. 2010), the TLS data provided a very dense coverage at the beach-face as well as at the swash zone, where foam and suspended matter reflect the laser signal. Further seaward, at the surf zone, distance measurements were obtained mostly from the breaking waves and propagating bores and the intermediate 'clear' water returned blank spots (Fig. 9). Even though there were no data available to validate the TLS measurements, visual inspection and comparison with the photographs confirmed previous studies showing that TLS can provide reliable hydrodynamic measurements. Unique in the present TLS application is the spatial coverage of the measurements, which can be compared only to the one of X-Band radars.

\section{SUMMARY AND CONCLUSIONS}

Field testing of RTS-Hanna showed that robotic platforms have very high potential to provide $\mathrm{cm}$ accuracy and $<0.5 \mathrm{~m}$ resolution topographic data from large coastal areas ( $>10 \mathrm{~km})$ in a time- and costefficient manner. Two days of testing including different runs with both manual and autonomous steering provided 175 GB of detailed topographic data, camera images, position and velocity information. All data were logged with a precise timestamp from the hard real-time operating system which is important for post-processing. Technical problems were identified and were mostly related to moving over loose sand, while solutions are available and are currently being evaluated. Firstly, wheel slippage occurred more often and severely than anticipated resulting in erroneous wheel odometry. This could be solved by either applying combined visual and laser/radar-based odometry for wheel slippage detection or by using a RTK-GPS, the latter of which being the more expensive solution. Secondly, driving at sand requires higher steering forces than typical on streets, in woodlands or on fields. The 
setup of the Paravan drive-by-wire system was not designed for such forces and in consequence had already been adapted to prevent steering forces from exceeding the specifications. Further improvements of the data logging could be made by using faster hardware.

RTS-Hanna could immediately be used under manual and even supervised autonomous operation which comprises an important step forward, given the amount of information collected by the calibrated sensor setup. Despite the obvious benefits, completely unsupervised autonomous operation would not be feasible in most countries due to legislation. Testing showed that one of the main safety issues in autonomous operation is to detect people lying on rough ground, which could be overcome by closing the measurement area for public use. Moreover, a typical problem in autonomous robotics is to distinguish weak obstacles, e.g. tall grass, which the robot could safely drive over and hard obstacles like metal poles which have to be avoided.

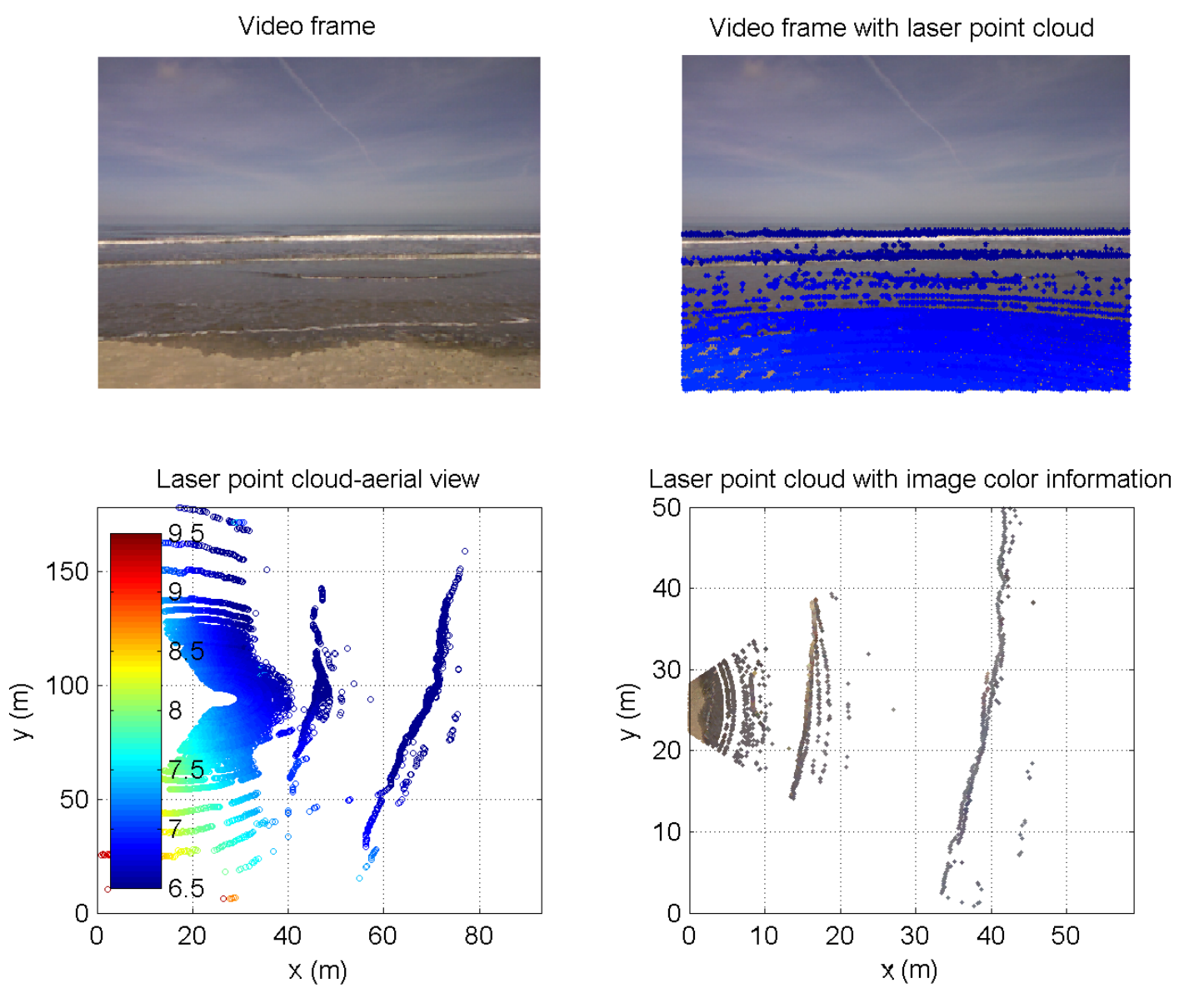

Figure 9. Water and beach surface elevation measurements at the surf/swash zone: Camera image (a) with superimposed scan data (b); scan points coloured by elevation (c, metrical scale) and by image colour (d). 


\section{FUTURE WORK}

There are a lot of possibilities for improvement in both hardware and software aspects of RTSHanna. Upgrading to a RTK-GPS would significantly improve the absolute position accuracy, as would a more sophisticated odometry fusing wheel encoder, laser and radar information. Even more detailed topographic data could be achieved by applying a very high resolution 3D laser scanner which in consequence would include the minor drawback of higher measurement durations. Moreover, further testing and validating the accuracy of the dry-beach and water elevation measurements under a wider range of cases would allow accessing the limitations and applicability of the TLS technique. Finally, real-time data logging and processing could become more efficient while the final data can comply with existing data-standards in order to facilitate post-processing with standard software.

Another important possible application of platforms like RTS-Hanna is to offer decision-support for coastal managers by providing real-time information about the beach morphological state and also about the spatial distribution of beach-users and infrastructure. Methodologies of automatic classification of beach objects and humans on the grounds of the TLS point clouds are currently under development, and will result in information about the beach-carrying capacity and the resulting demands which can be very useful to coastal managers.

\section{ACKNOWLEDGEMENTS}

The authors are grateful to Holger Blum from the NLWKN for the crucial help to solve all the administrative issues related to getting permission to drive a diesel-powered robot at Langeoog where usually electricity-powered vehicles are allowed only.

\section{REFERENCES}

Bleninsopp, C.E., M.A. Mole, I.L. Turner, and W.L. Peirson. 2010. Measurements of the time-varying free-surface profile across the swash zone obtained using an industrial LIDAR, Coastal Engineering, 57, 1059-1065.

De Vriend, H.J. 1991. Mathematical modelling and large scale coastal behaviour. Part I: physical processes, Journal of Hydrological Research, 29, 727-740.

Dellaert, F., D. Fox, W. Burgard, and S. Thrun. 1999. Monte carlo localization for mobile robots, Proceedings of International Conference on Robotics and Automation (ICRA1999), IEEE.

Ehlers, J. 1988. The Morphodynamics of the Wadden Sea. Balkema, Rotterdam, The Netherlands.

Flemming, B.W., and A. Bartholomä. 2003. Sedimentation and Erosion an der Nordseeküste, in: Lozán, J.L., E. Rachor, K. Reise, J. Sündermann, H.v. Westernhagen (Eds.), Warnsignale aus Nordsee \& Wattenmeer - Eine aktuelle Umweltbilanz, Wissenschaftliche Auswertungen/GEO, Hamburg pp. 57-60.

Folk, R.L..1980. Petrology of the Sedimentary Rocks. Hemphill Publishing Company, Austin, Texas, U.S.A.

Holman, R.A., and J. Stanley. 2007. The history and technical capabilities of Argus, Coastal Engineering, 54, 477-491.

Niemeyer, H.D. 1992. Die ursächliche Deutung von Transportphänomenen als Gestaltungsgrundlage für Strandauffüllungen. Die Küste, 54, 53-92.

Park, H.S., J.S. Sim, J. Yoo, and D.Y. Lee. 2011. Breaking wave measurement using Terrestrial LIDAR: validation with field experiment on the Mallipo Beach. Journal of Coastal Research, 64, 1718-1721.

Pietro, L.S., M.A. O'Neal, and J.A. Puleo. 2008. Developing terrestrial-lidar-based digital elevation models for monitoring beach nourishment performance, Journal of Coastal Research, 24, 15551564.

Senet, C.M., J. Seemann, S. Flampouris, and F. Ziemer, 2008. Determination of Bathymetric and Current Maps by the Method DiSC Based on the Analysis of Nautical X-Band Radar Image Sequences of the Sea Surface, Transactions on Geoscience and Remote Sensing, 46, 2267-2279.

Van Gaalen, J.F., S.E. Kruse, G. Coco, L. Collins, and T. Doering. 2011. Observations of beach cusp evolution at Melbourne Beach, Florida, USA, Geomorphology, 129, 131-140.

Vousdoukas, M.I., P.M. Ferreira, L.P. Almeida, G. Dodet, U. Andriolo, F. Psaros, R. Taborda, A.N. Silva, A.E. Ruano, and Ó Ferreira. 2011a. Performance of intertidal topography video monitoring of a meso-tidal reflective beach in South Portugal, Ocean Dynamics, 61, 1521-1540. 
Vousdoukas, M.I., G. Pennucci, R.A. Holman, and D.C. Conley. 2011b. A semi automatic technique for Rapid Environmental Assessment in the coastal zone using Small Unmanned Aerial Vehicles (SUAV), Journal of Coastal Research, 64, 1755-1759.

Wulf, O., C. Brenneke, and B. Wagner. 2004. Colored 2D Maps for Robot Navigation wit 3D Sensor Data, Proceedings of International Conference on Robots and Systems (IROS 2004), IEEE. 